\title{
Non-invasive method of field imaging in parallel plate waveguides
}

Iwaszczuk, Krzysztof; Andryieuski, Andrei; Lavrinenko, Andrei; Zhang, Xi-Cheng; Jepsen, Peter Uhd

Published in:

2011 36th International Conference on Infrared, Millimeter and Terahertz Waves (IRMMW-THz)

Link to article, DOI:

10.1109/irmmw-THz.2011.6105148

Publication date:

2011

Link back to DTU Orbit

Citation (APA):

Iwaszczuk, K., Andryieuski, A., Lavrinenko, A., Zhang, X-C., \& Jepsen, P. U. (2011). Non-invasive method of field imaging in parallel plate waveguides. In 201136 th International Conference on Infrared, Millimeter and Terahertz Waves (IRMMW-THz) IEEE. International Conference on Infrared, Millimeter and Terahertz Waves https://doi.org/10.1109/irmmw-THz.2011.6105148

\section{General rights}

Copyright and moral rights for the publications made accessible in the public portal are retained by the authors and/or other copyright owners and it is a condition of accessing publications that users recognise and abide by the legal requirements associated with these rights.

- Users may download and print one copy of any publication from the public portal for the purpose of private study or research.

- You may not further distribute the material or use it for any profit-making activity or commercial gain

- You may freely distribute the URL identifying the publication in the public portal 


\title{
Non-invasive method of field imaging in parallel plate waveguides
}

\author{
Krzysztof Iwaszczuk $^{\mathrm{a}}$, Andrei Andryieuski ${ }^{\mathrm{a}}$, Andrei Lavrinenko ${ }^{\mathrm{a}}$, Xi-Cheng Zhang ${ }^{\mathrm{b}}$, and Peter Uhd Jepsen ${ }^{\mathrm{a}}$ \\ ${ }^{a}$ DTU Fotonik, Technical University of Denmark, DK-2800 Kgs. Lyngby, Denmark \\ ${ }^{\mathrm{b}}$ Center for Terahertz Research, Rensselaer Polytechnic Institute, Troy, New York 12180, USA
}

\begin{abstract}
We present a new non-invasive air-photonic-based method of terahertz (THz) field imaging inside a parallel plate waveguide. The method is based on $\mathrm{THz}$ field-enhanced second harmonic generation of the fundamental laser beam in an external electric field. We also demonstrate the direct measurements of the frequency-dependent reflection coefficient at the end of the waveguide.
\end{abstract}

\section{INTRODUCTION}

$\mathrm{P}$ ARALLEL plate waveguide (PPWG) due to its simplicity but also unique properties has been an object of intensive investigation for guiding $\mathrm{THz}$ waves. The TEM mode of the PPWG is characterized by dispersionless propagation without a cut-off frequency, which makes it extremely useful for $\mathrm{THz}$ time domain spectroscopy (TDS) applications [1-3]. Recently tapered parallel plate waveguide (TPPWG) has been proposed [4-6]. It has been showed that TPPWG allows for subwavelength $\mathrm{THz}$ field confinement inside the TPPWG in both transverse dimensions [7], what could enable $\mathrm{THz}$ near-field imaging with subwavelength resolution. Also strong field enhancement can be achieved using TPPWG, which gives TPPWG big potential for investigation of nonlinear $\mathrm{THz}$ phenomena. For full experimental characterization of TPPWG properties, THz field distribution inside the waveguide is required. So far only method based on wave scattering from needle tip was demonstrated [8]. In this paper we present a new non-invasive method for characterization of the subpicosecond-long propagating electric transients inside a PPWG. The method is based on air biased coherent detection (ABCD) technique and does not disturb propagation of $\mathrm{THz}$ waves inside the waveguide. As an example of the application of our method a direct measurement of $\mathrm{THz}$ wave reflection from the waveguide end is presented.

\section{RESULTS}

The air biased coherent detection of $\mathrm{THz}$ radiation proposed by N. Karpowicz et al. [9] is based on the measurement of the terahertz-field-induced optical second harmonic generation (SHG) through a third order nonlinear process in the presence of external oscillating electric field $\mathrm{E}_{\text {bias. }}$ The intensity of the generated second harmonic $I_{2 \omega}$ measured using lock-in detection at the frequency of oscillating bias field is given by

$$
I_{2 \omega} \propto\left(\chi^{(3)} I_{\omega}\right)^{2} E_{\text {bias }} E_{T H z},
$$

where $\chi^{(3)}$ is the third-order susceptibility of air, $\mathrm{E}_{\mathrm{THz}}$ the electric field of the THz transient and $I_{\omega}$ is the intensity of the fundamental beam. In our case the fundamental beam of intensity $I_{\omega}$ propagates perpendicular to the waveguide and is focused in the volume between waveguide plates. The TPPWG consists of two electrically isolated, fine polished aluminum plates of a varying width (input $3 \mathrm{~mm}$, output 49 $\mu \mathrm{m}$ ) and a varying plate separation (input $1 \mathrm{~mm}$, output 200 $\mu \mathrm{m})$. The total length of the waveguide is $25.4 \mathrm{~mm}$. The metal plates of the TPPWG are connected to a high voltage modulator and in this way an external bias field $E_{\text {bias }}$ of \pm 5 $\mathrm{kV} / \mathrm{cm}$ at the point of the narrowest gap between metal plates is provided. Broadband $\mathrm{THz}$ pulses generated by tilted pulse front optical rectification in $\mathrm{LiNbO}^{2}$ [10] is coupled into the TPPWG at the wide end of the waveguide. The $\mathrm{THz}$ wave propagates between aluminum and then couples out into the free space at the narrow end of the waveguide. A near-infrared (NIR) beam from a $1 \mathrm{kHz}, 90 \mathrm{fs}, 800 \mathrm{~nm}$ regenerative Ti:sapphire femtosecond laser amplifier is used for probing the THz field between the plates of the waveguide. The NIR beam is focused in the center of the volume between the plates and recollimated after the waveguide. The 400-nm light generated in the ABCD process is measured by a photomultiplier tube (PMT) referenced to HVM modulation frequency. By moving the focus point of the fundamental beam along the waveguide and changing the time delay between the $\mathrm{THz}$ pulse and the probing pulse $\mathrm{THz}$ enhanced second harmonic can be mapped out at different position along the TPPWG.

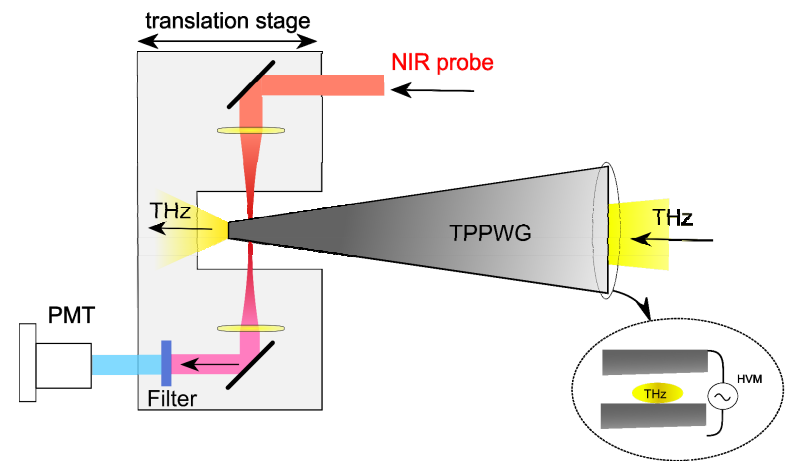

Fig. 1. Schematic of the air photonic setup for non-invasive field imaging inside a tapered parallel plate waveguide. $\mathrm{THz}$ radiation is coupled into the TPPWG, propagates along it and then interacts with NIR probe inducing second harmonic generation in the presence of oscillating local electric bias field delivered by high voltage modulator (HVM) and is measured by photomultiplying tube (PMT).

In Fig. 2 we show a $2 \mathrm{D}$ map of $\mathrm{THz}$ induced second harmonic $I_{2 \omega}(z, t)$ as a function of probe delay time $\tau$ for different positions $z$ along the waveguide. Two THz transients are present in the plot: the first transient, centered at 5 ps time delay, is the incident pulse propagating inside the waveguide. 
The second transient, propagating in the opposite direction, originates from the reflection at the waveguide end (position $\mathrm{z}$ $=0 \mathrm{~mm})$ due to the impedance mismatch between the waveguide $(\mathrm{z}<0)$ and free space $(\mathrm{z}>0)$. We have to note that for $z>0$ (outside the waveguide) the bias electric field quickly decays, which leads to vanishing $I_{2 \omega}(t, z)$.

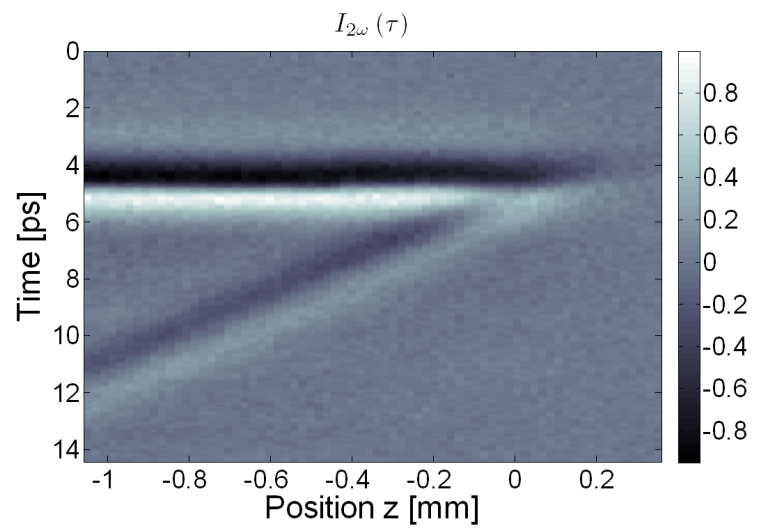

Fig. 2. 2D map of $\mathrm{THz}$ induced second harmonic $I_{2 \omega}$ as a function of probe delay time $\tau$ for different positions $z$ along the waveguide.

Fig. 3 presents measured frequency dependent amplitude refection coefficient of the waveguide defined as a ratio between Fourier components of reflected and incident electric fields. The reflection of the $\mathrm{THz}$ wave is caused by impedance mismatch between free space $Z_{0}$ and the waveguide $Z$ PPwG. We observe that the reflection coefficient $\Gamma=\left(Z_{\mathrm{PPWG}}-Z_{0}\right) /$ $\left(Z_{\text {PPWG }}+Z_{0}\right)$ decreases with increasing $\mathrm{THz}$ frequency, what is caused by frequency depended characteristics of ZPPWG. The data for extraction of $\Gamma(\mathrm{f})$ has been taken at a point $z=-0.9$ $\mathrm{mm}$ from the waveguide tip to assure good temporal separation between the incident and reflected transients. That implies that the calculated reflection coefficient $\Gamma$ includes not only reflection but also propagation effects, such as ohmic losses and wave scattering, which lead to additional reduction of the amplitude of the reflected wave.

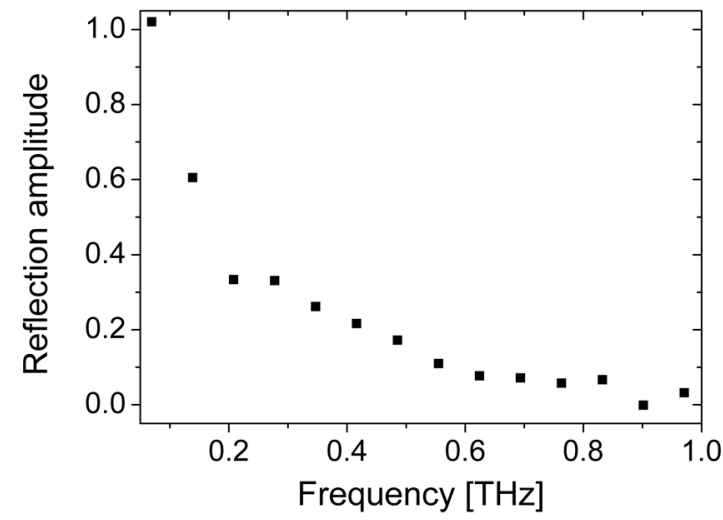

Fig. 3. Frequency dependent amplitude reflection coefficient $\Gamma(f)$ of the waveguide end.

In conclusion we have presented a new non-invasive technique of imaging broadband $\mathrm{THz}$ transients inside parallel plate waveguides. The method does not disturb the propagating $\mathrm{THz}$ pulses, what has been shown on the direct measurement of $\mathrm{THz}$ reflection coefficient from the end of the waveguide.

\section{REFERENCES}

[1] R. Mendis and D. Grischkowsky, "Undistorted guided-wave propagation of subpicosecond terahertz pulses," Opt. Lett. 26, 846 (2001).

[2] S. Coleman and D. Grischkowsky, "A THz transverse electromagnetic mode two-dimensional interconnect layer incorporating quasi-optics," Appl. Phys. Lett. 83, 3656 (2003).

[3] R. Mendis and D. M. Mittleman, "Comparison of the lowest-order transverseelectric (TE1) and transverse-magnetic (TEM) modes of the parallel-plate waveguide for terahertz pulse applications," Opt. Express 17, 14839 (2009)

[4] M. I. Stockman, "Nanofocusing of optical energy in tapered plasmonic waveguides," Phys. Rev. Lett. 93, 137404 (2004).

[5] A. Rusina, M. Durach, K. A. Nelson and M. I. Stockman, "Nanoconcentration of terahertz radiation in plasmonic waveguides," Opt. Express 16, 18576 (2008).

[6] H. Zhan, R. Mendis and D. M. Mittleman, "Superfocusing terahertz waves below $\lambda / 250$ using plasmonic parallel-plate waveguides," Opt. Express 18, 9643 (2010).

[7] H. Zhan, R. Mendis and D. M. Mittleman, "Characterization of the terahertz near-field output of parallel-plate waveguides," J. Opt. Soc. Am. B 28, 558 (2011).

[8] J. Liu, R. Mendis and D.M. Mittleman," The transition from a TEM-like mode to a plasmonic mode in parallel-plate waveguides," Appl. Phys. Lett. 98, 231113 (2011).

[9] N. Karpowicz, J. Dai, X. Lu, Y. Chen, M. Yamaguchi, H. Zhao, X.-C. Zhang, L. Zhang, C. Zhang, M. Price-Gallagher, C. Fletcher, O. Mamer, A. Lesimple and K. Johnson, "Coherent heterodyne time-domain spectrometry covering the entire "terahertz gap," Appl. Phys. Lett. 92, 011131 (2008)

[10] M. C. Hoffmann and J. A. Fulop, "Intense ultrashort terahertz pulses: generation and applications," J. Phys. D: Appl. Phys. 44, 08300 (2011) 